\title{
Construyendo la polarización a través del discurso político. Análisis del uso de claves periféricas en la retórica del presidente mexicano López Obrador ${ }^{1}$
}

Building polarization through political discourse. Analysis of the use of peripheral cues in the rhetoric of Mexican President López Obrador

\author{
CARLOS MUÑIZ \\ UNIVERSIDAD AUTÓNOMA DE NUEVO LEÓN ${ }^{2}$ \\ HTTPS://ORCID.ORG/0000-0002-9021-8198
}

Artículo recibido el / Article received: 2021-03-16

Artículo aceptado el / Article accepted: 2021-07-31

\begin{abstract}
RESUMEN: Desde su llegada a la presidencia de México en 2018, López Obrador ha establecido una estrategia comunicativa focalizada en sus conferencias de prensa diarias, denominadas mañaneras, a través de las que busca establecer la agenda informativa del país. Es habitual que en ellas el presidente utilice una retórica discursiva tendente a la polarización, para lo que se sirve del uso de claves o etiquetas que ofrecen un contexto para comprender los asuntos sobre los que se pronuncia. El artículo busca analizar el uso de etiquetas o claves por el presidente en su discurso, para lo que se realizó un análisis de contenido cuantitativo de una muestra de sus intervenciones $(N=867)$ durante un año en las mañaneras. Los hallazgos permitieron detectar dos esquemas discursivos a partir de la convergencia de diferentes claves: uno que tiende a presentar un Nosotros visualizado en un esquema de nuevo gobierno, vinculado con aspectos positivos como el compromiso o la lucha contra la corrupción, frente a un Otros que presenta al anterior gobierno, vinculado a aspectos negativos como la corrupción.
\end{abstract}

\footnotetext{
${ }^{1}$ El autor agradece a los colaboradores del proyecto por su apoyo en el desarrollo de la investigación a través de la codificación de las unidades de análisis y la categorización de las claves periféricas, así como a los revisores anónimos por sus valiosos comentarios.

${ }^{2}$ Este artículo fue desarrollado en el marco de un proyecto de investigación financiado por la Universidad Autónoma de Nuevo León (UANL-PAICYT) [clave CSA1159-20] y el Consejo Nacional de Ciencia y Tecnología (CONACYT) [clave 280739].
} 
Palabras clave: Discurso, clave periférica, etiqueta, polarización, López Obrador, México.

ABSTRACT: Since his arrival to the presidency of Mexico in 2018, López Obrador has established a communication strategy focused on his daily press conferences, called mañaneras, through which he tries to establish the informative agenda of the country. It is common for the president to use a discursive rhetoric tending to polarization, for which he makes use of cues or labels that offer a context to understand the issues on which he is pronounced. The paper seeks to analyze the use of labels or cues by the president in his discourse, for which a quantitative content analysis was carried out on a sample of his interventions $(N=867)$ during a year in the mañaneras. The findings made it possible to detect two discursive schemes from the convergence of different cues: one that tends to present an $U_{s}$ visualized in a new government scheme, linked to positive aspects such as engagement or the fight against corruption, compared to an Others that presents the previous government, linked to negative aspects such as corruption.

Key words: Discourse, peripheral cue, label, polarization, López Obrador, Mexico.

\section{INTRODUCCIÓN}

El pasado 1 de julio de 2018 México vivió la jornada electoral para la elección del nuevo presidente de la República, que supuso la victoria de Andrés Manuel López Obrador, al frente de una coalición electoral abiertamente vinculada con la izquierda (Martínez Garza \& Maltos, 2019). A partir de la llegada a la presidencia, López Obrador ha desarrollado una estrategia de comunicación social en la que destacan sobre otras actividades sus conferencias de prensa matutinas, denominadas mañaneras, con las que busca establecer la agenda informativa diaria (Chaguaceda, 2019; Villanueva Ulfgard \& Villanueva, 2020). En ellas, es habitual que las intervenciones del presidente presenten una fuerte carga ideológica que, en gran medida, busca la polarización a través del cuestionamiento permanente de los gobiernos anteriores y sus críticos actuales (Picazo Zamarripa et al., 2019; Sánchez Gudiño, 2020).

Para ello, se ha señalado que López Obrador se sirve de una narrativa discursiva que busca la confrontación a través de presentar la realidad política y social en forma de dos bandos -el de los buenos o nosotros y el de los malos o ellos (Marini, 2019; Moreno, 2019)-, que no contribuye tanto a justificar las acciones de gobierno sino más bien a mantener una campaña electoral permanente (Hernández Trejo, 2020) y generar una polarización de la sociedad (Picazo Zamarripa et al., 2019). Para ello, es habitual que promocione ciertos elementos retóricos, como encuadres o claves. En este sentido, se ha señalado que al construir el discurso se pueden utilizar ciertas claves o etiquetas, que acompañan a los argumentos centrales del mensaje para contextualizarlo y establecer una base para la interpretación y comprensión del asunto informado (Cho et al., 2006; Reese, 2007) mediante una ruta más simple o periférica con la que se impacta en la opinión pública a través de generar esquemas sencillos de comprensión.

Atendiendo a estos antecedentes, el presente artículo busca determinar el uso de etiquetas o claves periféricas dentro de la estrategia discursiva seguida por el presidente 
Andrés Manuel López Obrador para presentar una visión sobre la realidad política y social del país. En particular, se analizan sus intervenciones en la conferencia diaria mañanera, en tanto que constituye el principal ejercicio de establecimiento de agenda política realizada en la actualidad por el presidente, así como una plataforma para la transmisión de esquemas discursivos con los que el mandatario busca influir en la conformación de la opinión pública a través de un impacto a nivel cognitivo y afectivo.

\section{CONSTRUCCIÓN DEL DISCURSO POLÍTICO}

La relación especial existente entre los poderes públicos y actores políticos con los medios de comunicación es fuerte y está ampliamente estudiada, planteándose habitualmente que los primeros tienden a generar y/o mantener viva la información a la que los segundos dan cobertura (Lang \& Lang, 1981; Druckman, 2001). Una acción que viene determinada por las fuertes asociaciones existentes entre ambas instituciones, marcadas por una necesidad mutua, y en la que las élites constituyen una fuente informativa de primer orden, con legitimidad, fiabilidad e influencia. Esta relación, que constituye la base del proceso de creación de la agenda política, así como del tratamiento informativo ofrecido de los asuntos de actualidad (Rodelo \& Muñiz, 2019), supone para Mazzoleni (2010) una actitud deferente que el actor político tiene hacia la ciudadanía como respuesta a su deber de información y transparencia.

Para ello, los gobernantes y actores políticos se pueden apoyar en diferentes estrategias de comunicación, que constituyen la base del discurso político con el que se transmite información hacia los demás actores. Estos contenidos constituyen los media inputs, también llamados subsidios informativos, definidos por Rodelo y Muñiz (2019) como «todos aquellos materiales, como notas de prensa, conferencias de prensa, entrevistas y declaraciones, que los actores políticos realizan a las redacciones (de los medios) para facilitar su trabajo y, al mismo tiempo, influir sus encuadres noticiosos» (Rodelo \& Muñiz, 2019: 104). Estos subsidios constituyen el elemento esencial de la estrategia de comunicación realizada por los actores del sistema político, que se materializa en forma de comunicación pública e institucional, contacto personal o de propaganda y publicidad (Mazzoleni, 2010).

Ésta es la idea de los autores que han analizado y planteado la existencia de una relación entre las agendas política o de los partidos (policy o party agenda) y la mediática (media agenda), donde los actores políticos influirían en los medios de comunicación seleccionando y destacando o haciendo más salientes ciertos asuntos y temas que recibirán una mejor cobertura informativa (Lang \& Lang, 1981). Pero también la influencia que tienen las declaraciones oficiales de los gobernantes y actores políticos sobre los medios, donde los primeros buscan promover su punto de vista sobre un asunto o encuadre (advocacy frame) para influir en la elaboración de las noticias (Carragee \& Roefs, 2004; de Vreese, 2012; Matthes, 2012; Rodelo \& Muñiz, 2019). Ello lleva a que exista una fuerte competencia entre los diferentes actores políticos para controlar el establecimiento de la agenda política, pues en gran medida gracias a ello se puede incidir en las interpretaciones que la opinión pública hace de la realidad (Hänglli, 2012).

Sin embargo, la prominencia dentro de las fuentes políticas habitualmente se centra en las figuras gubernamentales, en especial los actores con la mayor responsabilidad dentro del mismo, como suelen ser el presidente en los sistemas presidenciales o el primer ministro en el caso de los regímenes parlamentarios (Glazier \& Boydstun, 2012; Rodelo \& Muñiz, 2019). Estos actores habitualmente tienen mayor capacidad de incidir en la agenda mediática, consiguiendo con ello además aumentar la frecuencia de presencia y prominencia de sus encuadres con los que ofrecen sus puntos 
de vista acerca de la realidad en los contenidos mediáticos que llegan a la ciudadanía (Hänglli, 2012; Muñiz, 2020). De esta manera, los diferentes actores del sistema político constituyen uno de los factores explicativos externos que determinan en gran medida la creación de encuadres o frame building que se realiza dentro de los medios (Scheufele, 2006; de Vreese, 2012).

Para ello, los actores políticos construyen y promueven ciertos frames en sus discursos políticos con los que buscan asentar sus puntos de vista sobre la realidad (Matthes, 2012). Estos encuadres han sido denominados de promoción (advocacy frame) (Carragee \& Roefs, 2004) y son aquellos que son «presentados por los diferentes participantes en un debate político» (de Vreese, 2012: 367). Por su parte, D'Angelo y Shaw (2018) los denominan encuadres de asunto (issue frame), señalando que son «desarrollados y expresados por individuos que construyen un argumento desde consideraciones, que son razones que favorecen un punto de vista de un asunto frente a otro posible» (D’Angelo \& Shaw, 2018: 211). Se observa, por tanto, que el framing constituye una poderosa herramienta a disposición de los actores políticos, que pueden emplear para la construcción de su discurso y la promoción de su visión acerca de la realidad (Hänglli, 2012; Matthes, 2012).

Normalmente son el presidente o el primer ministro los actores políticos con mayor capacidad de influencia, logrando introducir en el discurso público los encuadres más importantes, mismos que habitualmente serán los dominantes en la cobertura política ofrecida por los medios (Hänglli, 2012). Pero, junto a esta capacidad de influencia a través del framing, los actores políticos cuentan en su arsenal retórico con otros recursos para elaborar el discurso, como por ejemplo la categorización y etiquetado del asunto, es decir, la asignación de una etiqueta a los asuntos conocidos para influir en cómo son vistos por parte de los miembros de la opinión pública (Nelson, 2004). Al respecto, se ha detectado que la comprensión pública de los asuntos viene a menudo determinada por ciertas categorías o claves ofrecidas por las élites dentro de su discurso (Cho et al., 2006) y que acompañan al encuadre promovido dotándolo de una valencia favorable o desfavorable (D’Angelo \& Shaw, 2018).

\section{USO DE CLAVES Y ETIQUETAS PARA LA ELABORACIÓN DEL MENSAJE POLÍTICO}

No cabe duda de que cuando el actor político construye su discurso político busca, tanto de una manera indirecta a través de los medios de comunicación tradicionales como de una directa mediante el uso de los nuevos medios, influir las opiniones de la ciudadanía, modificando la elaboración de juicios y creencias, así como determinar la opinión pública. Para ello, el uso de ciertas herramientas en el mensaje será crucial, como puede ser la selección de asuntos, la promoción de encuadres o el uso de determinadas claves o etiquetas para ofrecer esquemas de interpretación a la audiencia que determinen las opiniones de los ciudadanos (Shah et al., 2002). No en vano se ha demostrado que el uso de claves periféricas, como pueden ser apelaciones al origen étnico o racial de un grupo social concreto o la credibilidad de la fuente informativa, tiene la capacidad de incrementar el efecto generado por los encuadres utilizados para presentar la información (Druckman, 2001; Igartua \& Cheng, 2009).

De esta manera, se asume que el uso de ciertas etiquetas o claves dentro del mensaje puede derivar en un procesamiento de la información por una vía periférica, que implica un escrutinio superficial del mensaje sin atender a los argumentos presentes en el mismo (Cho et al., 2006; Rotboim et al., 2019). Esto se vuelve crucial en el terreno político, pues es muy habitual que las estrategias de comunicación diseñadas dentro de 
las campañas impliquen el uso conjunto de encuadres y claves (Druckman et al., 2010), con las que se busca llegar a públicos diversos atendiendo a su capacidad y motivación para procesar la información. En el terreno periodístico, también es habitual que las noticias presenten de forma conjunta encuadres y claves para presentar la realidad, los cuales derivan en gran medida del intercambio de información existente entre las élites políticas y los periodistas y que constituye el proceso de construcción de los encuadres (Shah et al. 2002).

Así pues, se asume que al dotar de un contexto y un acercamiento a la realidad narrada en los mensajes, se suele utilizar un framing particular que se asocia con el uso de ciertas claves periféricas que acompañan la narración (Rotboim et al., 2019), que pueden operar como dispositivos del framing (D'Angelo \& Shaw, 2018). Al respecto, Reese (2007: 150) señala que es habitual que los frames en las noticias se presenten adornados con conceptos periféricos. Por ello, ambos elementos no deben ser confundidos pues juegan roles diferentes dentro del mensaje político (Cho et al., 2006). Así, mientras los frames son ideas organizadoras centrales del mensaje que tienden a poner de manifiesto patrones sólidos de argumentación a favor de ciertas posiciones sobre otras (Matthes, 2012), las claves o conceptos periféricos son más concretos y específicos y no es necesaria su presencia para que el mensaje se estructure y transmita una idea concreta (Hertog \& McLeod, 2001; Reese, 2007).

Esta diferencia entre claves y frames es también señalada por Cho et al. (2006), quienes marcan hasta tres características que los distinguen. Así, mientras que los encuadres se utilizan para estructurar globalmente el mensaje, las claves operan lingüísticamente definiendo objetos específicos. Esto lleva a que las claves sean particulares del tema u objeto abordado en el mensaje, mientras que los frames suelen ser recurrentes entre temas y objetos, sobre todo los que son genéricos. Finalmente, mientras que los encuadres derivan sobre todo de la práctica profesional periodística y de la reinterpretación de los encuadres promovidos por los actores políticos, las claves suelen derivar de la competencia ideológica que llevan a cabo las élites para caracterizar ciertas realidades. Todo ello lleva a los autores a definir las claves como «etiquetas utilizadas para identificar asuntos políticos, caracterizar grupos sociales y definir figuras políticas» en el mensaje (Cho et al., 2006: 136).

La visualización de estas claves lingüísticas (Edelman, 1993) o periféricas (Rotboim et al., 2019) dentro del mensaje se realiza a partir del uso de ciertas palabras. Al respecto, Northup et al. (2013: 3) señalan que las claves «consisten en palabras incrustadas en el texto destinadas a desencadenar los conceptos supuestamente relacionados». De esta manera, por ejemplo, el uso de la palabra inmigrante frente a ciudadano cambia radicalmente la manera como se interpreta la información acerca de ciertos grupos sociales. Esta práctica lleva a que exista una gran diversidad de clases de claves que pueden ser utilizadas para de estructurar el discurso como, por ejemplo, claves grupales, étnicas o raciales (Igartua \& Cheng, 2009; Northup et al., 2013), económicas (Shah et al., 2002) o ideológicas (Keum et al., 2005). A ello se unen las claves periféricas implícitas y/o explícitas (Valentino et al., 2002) que derivan del uso de recursos formales para construir el mensaje, como pueden ser ciertas características visuales (Druckman et al., 2010) o la apelación a expertos para transmitir el mensaje (Igartua \& Cheng, 2009).

Este ejercicio de etiquetado o categorización a través del uso de claves periféricas es muy habitual en el terreno político (Nelson, 2004), en tanto que constituyen potenciales armas ideológicas al servicio de los actores políticos para estructurar su discurso y establecer en la opinión pública ciertas creencias políticas y emociones, como la alegría, el entusiasmo o el miedo, hacia la realidad que les rodea (Edelman, 1993). Pero, debido a su carga ideológica, también pueden contribuir al establecimiento de antagonismos, 
pues mediante la utilización de palabras se construyen poderosas metáforas que evocan marcos o contextos de interpretación discutibles, y no tanto descripciones factuales sobre la realidad existente (Edelman, 1993; Cho et al., 2006). La utilización en el lenguaje de expresiones como derecha, izquierda, conservador o liberal, por poner algunos ejemplos, evoca imágenes en la mente que, dentro de cierto patrón de creencias ideológicas, pueden llevar a que algo se visualice como respetable o reprobable (Edelman, 1993; Acree et al., 2020).

La efectividad de las claves para establecer estos patrones de comprensión en la mente depende en gran medida de su nivel de complejidad; conforme más sencillas y entendibles sean las palabras, mayor será su efectividad (Cho et al., 2006). Además, si su inserción se realiza de forma natural en el mensaje, mediante expresiones utilizadas coloquialmente por la audiencia y no tanto con etiquetas construidas como herramienta de propaganda, su utilidad tiende a ser mayor (Edelman, 1993). También se ha señalado que su resonancia es mayor cuando aparecen en conjunto con otras claves, logrando mayores reacciones entre los miembros de la audiencia (Cho et al., 2006; Igartua \& Cheng, 2009). De hecho, es normal que en los discursos exista esta convergencia de claves (Northup et al., 2013; Cho et al., 2006), mediante la presentación conjunta de diferentes detonadores que en el terreno político pueden llevar a crear categorías ideológicas, como las de derecha o izquierda que deriven en una polarización de la audiencia (Acree et al., 2020).

\section{CONTEXTO DE ESTUDIO: ESTRATEGIA DISCURSIVA DE LÓPEZ OBRADOR}

El proceso electoral mexicano que culminó con la elección realizada el 1 de julio de 2018 destacó por tratarse de la elección más grande en la historia del país, con un total de 3400 cargos de elección que se pusieron en juego (Esteinou, 2019). Pero, sin duda la característica más destacable de este proceso fue el triunfo y elección como presidente de México de Andrés Manuel López Obrador al tratarse de un candidato abiertamente vinculado a la izquierda y que rompía con el poder de los partidos tradicionales (Martínez Garza \& Maltos, 2019). Su elección vino acompañada del triunfo de la coalición electoral Juntos Haremos Historia y, en especial, del partido político Morena (Movimiento Regeneración Nacional) liderado por el candidato presidencial, en numerosos de los cargos sometidos a elección, lo que ha llevado a calificar la victoria de López Obrador como un auténtico tsunami dentro del sistema político del país (Esteinou, 2019).

Un triunfo que se gestó, en gran medida, gracias a una campaña de comunicación permanente que López Obrador llevaba haciendo desde su primera candidatura a la presidencia en 2006, que le permitió contar con un nivel de conocimiento ciudadano muy elevado. Con todo, no cabe duda de que su triunfo también se vio favorecido por el contexto político existente en el país, marcado en gran medida por décadas de enojo, desilusión y desafección ciudadana con la clase política y una crisis del sistema político e institucional mexicano que se vio intensificada en los años previos a la elección de 2018 (Esteinou, 2019; Marini, 2019). A partir del inicio de su gestión, el presidente ha establecido una estrategia de comunicación social de corte personalista, que rompió con los modelos mantenidos por anteriores gobiernos, al basarse en gran medida en su propio liderazgo y en una relación de tipo unidireccional con los medios (Picazo Zamarripa et al., 2019; Sánchez Gudiño, 2020).

Dentro de los media inputs o subsidios informativos (Rodelo y Muñiz, 2019) que realiza López Obrador, destacan sobre otras actividades sus conferencias de prensa matutinas que el presidente efectúa diariamente y que son conocidas como mañaneras 
(Esteinou, 2019). En ellas, el presidente establece su posición política, así como la de su gobierno, acerca de los asuntos públicos relevantes para el país y de las políticas públicas impulsadas desde el gobierno. Una herramienta, por tanto, crucial con la que López Obrador busca en gran medida establecer la agenda informativa diaria del país, así como la promoción de encuadres para presentar a la opinión pública puntos de vista acerca de la realidad social (Chaguaceda, 2019; Villanueva Ulfgard \& Villanueva, 2020). Pero, más allá de su función puramente informativa, las mañaneras también cumplen una labor política, siendo habitual que en ellas el presidente cuestione a sus críticos y a los anteriores gobiernos (Sánchez Gudiño, 2020), buscando con ello «mantener un control político» (Picazo Zamarripa et al., 2019: 141).

En este sentido, desde el inicio de su administración en 2018, el presidente ha buscado permanentemente en sus discursos establecer una distinción respecto de los gobiernos anteriores, la denominada habitualmente como época neoliberal con la que su cuarta transformación supone una ruptura total (Sánchez Talanquer, 2020). Para ello, el mandatario se suele apoyar en un estilo retórico cargado de afirmaciones antagonistas (Amezcua Yépiz, 2019; Villanueva Ulfgard \& Villanueva, 2019), con una tendencia a presentar la realidad política y social mexicana mediante una narrativa discursiva en la que se presentan dos bandos, el de los buenos o nosotros y el de los malos o ellos (Marini, 2019; Moreno, 2019). Una narrativa discursiva que para Sánchez Talanquer (2020: 422) implica la utilización de «una retórica hostil» de oposición a los adversarios y que busca la polarización de la sociedad (Picazo Zamarripa et al., 2019).

En este sentido, se ha señalado que más que buscar informar y justificar las acciones y de gobierno, parece tratarse más bien de un ejercicio discursivo con el que busca mantener una campaña electoral permanente (Picazo Zamarripa et al., 2019; Hernández Trejo, 2020), utilizando un discurso polarizado ideológicamente con expresiones que habitualmente forman parte de la retórica electoral. Para esto, hace uso de un discurso marcado por claves con fuerte carga ideológica, pero que son sencillas y fáciles de asimilar por un público general (Picazo Zamarripa et al., 2019) y que se han detectado como las más efectivas a la hora de impactar sobre la opinión pública (Cho et al., 2006). Es el caso de expresiones como fifí, empleado para aludir a la élite política (Amezcua Yépiz, 2019; Villanueva Ulfgard \& Villanueva, 2019) o a sus opositores y gobiernos anteriores, que también suelen ser englobados dentro de una mafia del poder (Picazo Zamarripa et al., 2019) vinculada con corrupción o el conservadurismo (Marini, 2019; Sánchez Talanquer, 2020).

Tomando en consideración estos antecedentes y contexto de estudio, el artículo plantea las siguientes preguntas de investigación a las que se espera dar respuesta:

PI1: ¿Cuáles son las etiquetas o claves periféricas más utilizadas habitualmente por el presidente López Obrador en sus conferencias matutinas?

PI2: ¿Existe una convergencia entre las claves periféricas o etiquetas que permita visualizar los esquemas discursivos utilizados por el presidente para construir su discurso político?

PI3: ¿Qué esquemas discursivos utiliza el presidente en su discurso político?

\section{MÉTODO SEGUIDO}

\subsection{MUESTRA Y UNIDAD DE ANÁLISIS}

Tomando en consideración las preguntas de investigación planteadas, se realizó un análisis de contenido de corte cuantitativo de las intervenciones del presidente López 
Obrador en su conferencia de prensa denominada mañanera que se realiza diariamente. Para ello, se determinó una muestra representativa de las mañaneras del presidente a partir de la selección de cinco días mensuales que permitieran determinar una semana construida de forma aleatoria para cada mes durante un año (julio de 2019 a junio de 2020). Dentro de las 60 mañaneras seleccionadas, se detectaron todas las intervenciones realizadas por el presidente López Obrador a partir de la revisión de las grabaciones de las conferencias que están disponibles en YouTube. Esta búsqueda resultó en una muestra final de 867 intervenciones que fueron codificadas como unidades de análisis, con una duración en promedio de 4:31 minutos ( $D E=6: 28)$ para el conjunto de las intervenciones.

En primer lugar, se realizó una inmersión cualitativa para tratar de ubicar en las intervenciones todas las claves o etiquetas utilizadas por el presidente en su discurso, para lo que se trabajó con una submuestra de 12 mañaneras elegidas al azar. El trabajo de revisión fue realizado por cuatro codificadores que reportaron por separado todas las claves encontradas. A partir de este documento, un analista realizó una codificación abierta de todas las claves detectadas para determinar, a partir de un acercamiento inductivo, las categorías existentes siguiendo un criterio de similitud semántica. Este proceso resultó en la detección de 28 categorías generales que agrupaban un número elevado de etiquetas y/o claves que presentaban un alto grado de semejanza entre ellas.

Para dotar a este proceso de confiabilidad, un segundo analista procedió a realizar el mismo ejercicio de categorización que supuso la detección de 16 grupos de claves de forma diferenciada. A partir de la comparación entre las categorías de ambos analistas, se pudieron detectar semejanzas en al menos 15 de los conjuntos de etiquetas o claves para el listado generado por cada analista, tomando en consideración que algunos de los conjuntos propuestos por el analista segundo englobaban varios de los que fueron localizados por el primer analista (Ver Tabla 1). De esta manera, el criterio utilizado para la selección final de las categorías sometidas a análisis no dependió de la voluntad de un único investigador, sino más bien del acuerdo intersubjetivo entre ambos analistas, lo que validó el proceso de creación de las variables. Por ello, se decidió trabajar en adelante únicamente con estas categorías generales, descartando las demás propuestas por cada analista de forma separada.

Tabla 1. Comparativa de categorías o grupos de claves detectadas por ambos analistas para elaborar el libro de códigos.

\begin{tabular}{|l|l|}
\hline \multicolumn{1}{|c|}{ Analista 1 } & \multicolumn{1}{c|}{ Analista 2 } \\
\hline - Anterior gobierno & • Antiguo régimen \\
\hline - Conservadores & • Conservadores \\
\hline - Privilegiados & - Corrupto o corruptos \\
\hline - Corruptos & \\
\hline - Privatización & - Prácticas de corrupción \\
\hline - Tecnócratas & - Neoliberales \\
\hline - Neoliberalismo & - Neoliberalismo \\
\hline - Corrupción & - Corrupción \\
\hline - Oposición & - Políticos disidentes \\
\hline - Actual gobierno & - Cuarta transformación \\
\hline - Compromiso & - Prácticas de la cuarta transformación \\
- Política diferente & \\
- Lucha contra la corrupción & \\
\hline - Respeto a la ley & \\
\hline - El pueblo & Pueblo \\
\hline
\end{tabular}




\subsection{LIBRO DE CÓDIGOS}

Por tanto, el libro de códigos utilizado para este artículo quedó compuesto por las 15 variables que reflejaban las etiquetas o claves utilizadas por el presidente para organizar su discurso, a partir de las categorías detectadas de forma inductiva en el ejercicio cualitativo realizado previamente a la codificación cuantitativa. Para cada una de ellas se evaluó su presencia (1) o no (0) en el discurso. A continuación, se expone de forma breve cada una de las categorías o grupos de claves debido a que en algunas fueron muchas las expresiones que las conformaban:

1) Anterior gobierno: Define el anterior gobierno como antiguo régimen, élite, los que mandaban, lo que ya no queremos, un elefante reumático y mañoso, que hacía política nefasta, bueno para nada, etcétera.

2) Conservadores: Se refiere a otros políticos como conservadores, charro conservador, el conservadurismo, etcétera.

3) Privilegiados: Se refiere a otros políticos como los acostumbrados a tener lambiscones, barberos, aplaudidores del gobierno, a quemarle incienso, a tener sueldos elevadísimos, colmados de atenciones y privilegios, etcétera.

4) Oposición: Se refiere a otros políticos como enemigos, adversarios, quienes se oponen, quienes están en contra, opositores, la oposición, etcétera.

5) Corruptos: Se refiere a otras personas como corruptos, ladrones, alcahuetes del régimen de corrupción, defraudadores, influyentes, ambiciosos, minoría rapaz, quienes no actúan con ética, traficantes de influencia, etcétera.

6) Neoliberalismo: Se refiere a la anterior etapa como periodo neoliberal, de la política neoliberal, el sistema neoliberal, neoliberalismo, neoporfirismo, modelo neoliberal mundial, gobiernos neoliberales, etcétera.

7) Tecnócratas: Se refiere a políticos anteriores como tecnócratas aplaudidos, afamados, eminencias, expertos, financieros, de tener una visión tecnocrática, etcétera.

8) Corrupción: Habla de la corrupción, contubernio o complicidad entre autoridades, mordidas, influyentismo, oportunidad para enriquecerse, condonación de impuestos, condonación de impuestos, abusos, extorsiones, se robaba, etcétera.

9) Privatización: Se refiere a la actividad de anteriores gobernantes como actos de privatización, privatizar, convertir lo público en privado, entregar la educación al mercado, convertir todo en una mercancía, etcétera.

10) Actual gobierno: Al hablar del nuevo gobierno, lo define como un nuevo paradigma, nueva realidad, cambio, estrategia nueva, otra política, distintos, nueva etapa, gobernar escuchando el mandato, transformación de México, Cuarta Transformación, etcétera.

11) Compromiso: Define su actuación y de su gobierno como un reto, desafío que están enfrentando, esfuerzo, compromiso para mejorar diferentes aspectos, etcétera.

12) Política diferente: Al comparar con la anterior política señala que ahora no es igual, se actúa con rectitud, con apego a la verdad, con cero impunidad, haciendo cambios, reformas, limpiando, recuperando lo perdido, etcétera.

13) Lucha contra la corrupción: Habla de acabar con la corrupción, no permitirla, enfrentarla, combatirla, no tolerarla, de que haya cero corrupción, etcétera.

14) Respeto a la ley: Habla de respetabilidad, ser respetuosos de los derechos, la responsabilidad, transparentar, garantizar libertades, etcétera.

15) El pueblo: Menciona a la gente, el pueblo, todos los mexicanos, el pueblo de México, nuestro pueblo, los mexicanos, la mayoría de los mexicanos, etcétera. 


\subsection{CODIFICACIÓN Y FIABILIDAD DEL ESTUDIO}

En la codificación y grabación de las unidades de análisis participaron cuatro estudiantes de licenciatura que realizaron una estancia de verano científico de forma virtual en el Laboratorio de Comunicación Política (LACOP) de la Facultad de Ciencias Políticas y Relaciones Internacionales de la Universidad Autónoma de Nuevo León. Como paso previo a la codificación, todos recibieron capacitación en el uso del libro de códigos elaborado y se realizó una prueba de entrenamiento con unidades elegidas al azar. Una vez concluido el trabajo de codificación, se hizo un nuevo análisis con una submuestra $(n=53)$ para realizar un chequeo inter-jueces que arrojó un valor .89 atendiendo a la fórmula acuerdo de Holsti en promedio para las variables. Tomando en consideración las condiciones en que se realizó el estudio, de forma virtual debido a la afectación de la pandemia de COVID-19, se considera que los datos son aceptables y fiables, pudiéndose proceder al análisis de los resultados.

\section{ANÁLISIS DE LOS RESULTADOS}

Tabla 2. Análisis descriptivo de las categorías o grupos de claves periféricas detectadas en los discursos.

\begin{tabular}{|l|c|c|c|c|c|}
\hline & Mín. & Máx. & $M$ & $D E$ & $\%$ \\
\hline Anterior gobierno & 0 & 1 & .19 & .39 & 18.9 \\
\hline Conservadores & 0 & 1 & .07 & .26 & 7.5 \\
\hline Privilegiados & 0 & 1 & .07 & .25 & 6.8 \\
\hline Oposición & 0 & 1 & .07 & .26 & 7.2 \\
\hline Corruptos & 0 & 1 & .15 & .36 & 14.9 \\
\hline Neoliberalismo & 0 & 1 & .09 & .29 & 9.1 \\
\hline Tecnócratas & 0 & 1 & .03 & .18 & 3.2 \\
\hline Corrupción & 0 & 1 & .19 & .40 & 19.4 \\
\hline Privatización & 0 & 1 & .03 & .18 & 3.2 \\
\hline Actual gobierno & 0 & 1 & .21 & .41 & 20.8 \\
\hline Compromiso & 0 & 1 & .20 & .40 & 20.4 \\
\hline Política diferente & 0 & 1 & .17 & .37 & 16.8 \\
\hline Lucha contra la corrupción & 0 & 1 & .14 & .34 & 13.7 \\
\hline Respeto a la ley & 0 & 1 & .12 & .32 & 11.9 \\
\hline El pueblo & 0 & 1 & .18 & .39 & 18.1 \\
\hline
\end{tabular}

Nota: En el análisis se utiliza el listado global de etiquetas o claves periféricas detectadas en el acercamiento cualitativo a los discursos, operacionalizadas como variables en el estudio. $M=$ Media; $D E=$ Desviación Estándar. El porcentaje se calcula con relación al número de unidades donde se presentaron las etiquetas.

El análisis descriptivo de las variables utilizadas en el estudio (Ver Tabla 2) permitió determinar que las claves periféricas o etiquetas detectadas en el discurso presidencial en mayor medida fueron las que hacían referencia al gobierno actual del país $(M=.21, D E=.41)$, con una presencia en el $20.8 \%$ de las intervenciones, así como la etiqueta referida a compromiso $(M=.20, D E=.40)$ con una aparición en el $20.4 \%$ de las intervenciones. Por otra parte, también tuvieron una presencia importante las claves periféricas referidas a la corrupción $(M=.19, D E=.40)$, presente en el $19.4 \%$ de las intervenciones, así como las referencias al anterior gobierno $(M=.19, D E=.39)$ que aparecieron en el $18.9 \%$ de las intervenciones. Finalmente, también destacó la referida a el pueblo $(M=.18, D E=.39)$ con una aparición en el $18.1 \%$ de las intervenciones. Se realizó un análisis de correlaciones entre todas las variables para determinar si había 
colinealidad entre ellas, es decir, si se presentaba un problema de redundancia de la información contenida en los análisis derivada del hecho de que algunas de las variables pudieran llegar a considerarse iguales entre sí (Igartua, 2006). Al detectarse que la asociación más fuerte fue la existente entre las etiquetas de corrupción y corruptos, $r(865)$ $=.55, p<.001$, se pudo descartar la existencia de colinealidad, lo que permitió seguir adelante con los análisis multivariados previstos.

Atendiendo a la segunda pregunta de investigación planteada en el estudio, el siguiente paso dentro del análisis de datos consistió en determinar la organización interna existente entre las 15 claves periféricas o etiquetas analizadas, para determinar si se agrupaban entre sí conformando una estructura latente en forma de factores que permitieran visualizar los esquemas utilizados por el presidente en su estrategia discursiva seguida en sus conferencias mañaneras. Para ello, se realizó un análisis factorial de componentes principales, con rotación ortogonal varimax, para determinar la validez estructural de los diferentes reactivos evaluados, una técnica de análisis multivariado que permite «examinar como se agrupan entre sí un grupo de variables, en función de su grado de correlación, con el propósito de descubrir si comparten alguna estructura latente» (Igartua, 2006: 628). El análisis arrojó un total de cuatro factores o dimensiones que en conjunto explicaban el $52.50 \%$ de la varianza (Ver Tabla 3). La calidad del análisis factorial se comprobó mediante el estadístico Kaiser-Meyer-Olkin (KMO) que arrojó un valor de .86 lo que señala una adecuación meritoria del análisis factorial. Además, el contraste de esfericidad de Bartlett resultó estadísticamente significativo, $\chi^{2}(105, N=$ $867)=2814.013, p<.001$, lo que permite determinar la correlación entre las variables, que en ningún caso llegaron a presentar problemas de colinealidad, como ya fue señalado con anterioridad.

Tabla 3. Resultados del primer análisis factorial de las categorías o grupos de claves periféricas detectadas en los discursos.

\begin{tabular}{|l|c|c|c|c|}
\hline \multicolumn{1}{|c|}{ Categorías } & \multicolumn{4}{c|}{ Factores } \\
\cline { 2 - 5 } & 1 & 2 & 3 & 4 \\
\hline Corruptos & .78 & & & \\
\hline Corrupción & .74 & & & \\
\hline Anterior gobierno & .63 & & & \\
\hline Lucha contra la corrupción & .59 & .37 & & \\
\hline Privilegiados & .52 & & & \\
\hline Privatización & .48 & & & -.36 \\
\hline Compromiso & .16 & .71 & & \\
\hline Actual gobierno & .38 & .66 & & \\
\hline Respeto a la ley & & .61 & .43 & \\
\hline Política diferente & & .61 & & \\
\hline El pueblo & & .60 & & \\
\hline Opposición & & & .80 & \\
\hline Conservadores & & & .60 & \\
\hline Tecnócratas & & & & .79 \\
\hline Neoliberalismo & .37 & & & .51 \\
\hline
\end{tabular}

Nota: En el análisis se utiliza el listado de etiquetas o claves periféricas detectadas en el acercamiento cualitativo a los discursos, operacionalizadas como variables en el estudio.

El primer componente extraído a partir del análisis factorial quedó configurado por ocho ítems que explicaban en conjunto el $19.51 \%$ de la varianza $(\alpha=.79)$, mientras que el segundo componente se configuró a partir de seis ítems que explicaban el $14.48 \%$ 
de la varianza $(\alpha=.74)$. Por su parte, el tercer componente estaba compuesto por tres reactivos $(\alpha=.41)$ que explicaban el $9.96 \%$ de la varianza y el cuarto componente por tres ítems $(\alpha=.30)$ que explicaban el $8.18 \%$ de la varianza. Atendiendo a que la consistencia interna de los dos últimos componentes era baja $(\alpha<.70)$, es decir, las diferentes variables que los integraban no llegaban a medir «una misma característica o atributo» (Igartua, 2006: 656), se procedió a eliminar aquellos ítems que únicamente tuvieran un peso factorial fuerte dentro de estos dos componentes. Con los 12 reactivos restantes se procedió a realizar un nuevo análisis factorial de componentes principales, con rotación ortogonal varimax, que convergió en dos dimensiones que en conjunto explicaban el $44.80 \%$ de la varianza (Ver Tabla 4). El estadístico Kaiser-Meyer-Olkin $(\mathrm{KMO}=.86)$ y el contraste de esfericidad de Bartlett, $\chi^{2}(66, N=867)=2488.91, p<$ .001 , fueron meritorios.

Tabla 4. Resultados del segundo análisis factorial de las categorías o grupos de claves periféricas detectadas en los discursos.

\begin{tabular}{|l|c|c|}
\hline \multirow{2}{*}{\multicolumn{1}{|c|}{ Categorías }} & \multicolumn{2}{c|}{ Factores } \\
\cline { 2 - 3 } & 1 & 2 \\
\hline Corruptos & .81 & \\
\hline Corrupción & .77 & \\
\hline Anterior gobierno & .63 & \\
\hline Lucha contra la corrupción & .63 & .34 \\
\hline Privilegiados & .54 & \\
\hline Neoliberalismo & .51 & \\
\hline Privatización & .39 & \\
\hline Compromiso & & .71 \\
\hline Actual gobierno & .38 & .65 \\
\hline Respeto a la ley & & .63 \\
\hline El pueblo & & .62 \\
\hline Política diferente & .34 & .60 \\
\hline
\end{tabular}

Nota: En el análisis se utilizan como variables las etiquetas o claves periféricas que convergieron en los dos principales factores detectados en el primer análisis factorial realizado.

Una vez detectados los dos factores extraídos a partir de este segundo análisis factorial, se procedió a calcular la validez interna de cada uno de esos componentes para determinar su fiabilidad o consistencia interna y poder así depurar las escalas, para lo que se utilizó la prueba Alpha de Cronbach. En cuanto al primer factor, se observó que el mismo quedaba compuesto por nueve ítems que explicaban en conjunto el $25.48 \%$ de la varianza $\left(\alpha_{\text {inicial }}=.81\right)$, mientras que el segundo componente quedó configurado por seis reactivos que explicaban en su conjunto el $19.32 \%$ de la varianza $\left(\alpha_{\text {inicial }}=.74\right)$. Atendiendo a que ambos factores presentaban buena consistencia interna, pero que había tres reactivos con peso factorial fuerte en ambos componentes, se analizó la incidencia que tenía su eliminación en cada uno de los factores para decidir en cuál de los dos se mantendrían. Un nuevo análisis de la consistencia interna, pero esta vez a través del procedimiento de eliminación de ítems, permitió determinar que era más conveniente mantener los tres reactivos en el segundo factor en detrimento del primero.

En cuanto el primer componente o esquema discursivo, que fue denominado como Ellos, quedó constituido por un conjunto de seis claves periféricas o etiquetas utilizadas en el discurso por el presidente para referirse a la oposición y anteriores administraciones a partir del uso conjunto de claves periféricas como anterior gobierno, corruptos, corrupción, privilegiados, neoliberalismo y/o privatización. Este esquema estaba 
presente en al menos el $33.5 \%$ de las intervenciones del presidente $(M=.12, D E=.21$, $\left.\alpha_{\text {final }}=.73\right)$. Por su parte, el segundo componente o esquema discursivo, que fue denominado como Nosotros, quedó constituido por la suma de seis claves o etiquetas presentes en el discurso que hacían referencia a la presencia en el discurso del presidente de etiquetas referidas al actual gobierno, vinculándolo con claves periféricas como la de compromiso, respeto a la ley, el pueblo, política diferente y/o lucha contra la corrupción. En este caso, el esquema estaba presente en al menos el $44.8 \%$ de las intervenciones del presidente $\left(M=.17, D E=.25, \alpha_{\text {final }}=.74\right)$.

A partir del análisis comparativo entre ambas variables se pudo determinar que el uso del esquema discursivo referido al Nosotros estaba, en términos generales, más presente en la retórica discursiva del presidente que el esquema de Ellos, $t(866)=-6.616$, $p<.001, d=0.22$. Para ello, se calculó una prueba $t$ de Student de muestras relacionadas, aplicable cuando la comparación se realiza «en base a un criterio propio de los sujetos» (Igartua, 2006: 550), en este caso dos variables procedentes de la misma muestra de intervenciones. En todo caso, las diferencias entre ambos esquemas no fueron muy fuertes, atendiendo al resultado relativo al tamaño del efecto. Ello se relaciona además con el hecho de que había una fuerte covarianza entre ambos esquemas, $r(865)=.55, p<$ .001 , lo que remite a una estrategia discursiva que tiende a enfatizar ambos esquemas de forma conjunta en el discurso. Con todo, tan sólo se detectó un $24 \%$ de intervenciones donde se hacía un uso conjunto de ambos esquemas con al menos la utilización de una clave para cada componente, lo que permite observar que, a pesar de ser los esquemas dominantes, en el discurso presidencial se presentaron otras claves periféricas que quedaron fuera de estos conjuntos de etiquetas detectados.

\section{DISCUSIÓN Y CONCLUSIONES}

Este artículo se planteó como objetivo determinar el uso de etiquetas o claves periféricas realizado por el presidente mexicano López Obrador en sus conferencias mañaneras, principal herramienta discursiva utilizada dentro de su estrategia de comunicación para presentar una visión sobre la realidad política y social del país a la opinión pública. En este sentido, el trabajo se centró en el análisis de los elementos que se presentan de forma adyacente a los argumentos en el discurso, en forma de claves periféricas o etiquetas que tienen la capacidad de incidir en la interpretación y comprensión de los asuntos a través de «identificar asuntos políticos, caracterizar grupos sociales y definir figuras políticas» en el mensaje (Cho et al., 2006: 136). Tomando como referencia este objetivo, la primera pregunta de investigación cuestionaba cuáles eran las etiquetas o claves periféricas más utilizadas habitualmente por el presidente López Obrador en sus conferencias matutinas.

El análisis cualitativo previamente realizado permitió detectar la presencia de hasta 15 claves periféricas o etiquetas que tendían a estar presentes en los discursos del presidente. Sin embargo, el acercamiento cuantitativo reflejó cómo la referencia en sus intervenciones al gobierno actual del país, mediante expresiones como nueva realidad, cambio o Cuarta Transformación, así como al compromiso, mediante expresiones como reto, desafío o esfuerzo, fueron las etiquetas más utilizadas. En consonancia con estudios previos, también se detectó un fuerte uso de la etiqueta corrupción (Marini, 2019; Sánchez Talanquer, 2020) y las expresiones a ella asociadas, como complicidad entre autoridades, mordidas, influyentismo o abuso. Finalmente, también destacó la utilización de expresiones vinculadas a los anteriores gobiernos, que son presentados como el antiguo régimen, los que mandaban o buenos para nada, vinculados a esa mafia del poder con que suele referirse a los críticos y opositores (Picazo Zamarripa et al., 2019). 
Se observa, por tanto, que en el discurso realizado por el presidente tienden a presentarse ciertas claves periféricas o etiquetas que, siguiendo lo planteado por autores como Cho et al. (2006) o Northup et al. (2013), suelen converger dentro del mensaje. En consonancia con ello, se planteó la segunda pregunta de investigación, que cuestionaba si se presentó esta convergencia entre claves periféricas o etiquetas que permitiera visualizar ciertos esquemas discursivos utilizados por el presidente para construir su discurso político. Los resultados del análisis realizado pusieron de manifiesto que las claves periféricas se organizaban entre sí, dejando entrever la existencia de una estrategia tendente a generar ciertos esquemas discursivos a través de la convergencia entre diferentes etiquetas que se posicionan conjuntamente para tratar de influir en mayor medida en la configuración de las actitudes y creencias de la ciudadanía acerca de la realidad social y política existente en el país.

Además, y en consonancia con la tercera pregunta de investigación, los resultados del estudio realizado ponen de manifiesto la utilización por parte del presidente López Obrador de una narrativa discursiva que se apoya en la utilización de dos esquemas discursivos antagónicos, construidos mediante la convergencia de diferentes claves periféricas con fuerte carga ideológica. Por una parte, en su discurso habitualmente se presenta la categoría de Nosotros visualizada en un esquema de nuevo gobierno que es sistemáticamente vinculado con aspectos positivos, además de constantes apelaciones al pueblo. Por otra parte, utiliza la categoría de Ellos que se visualiza a través de un esquema donde los anteriores gobiernos son vinculados con aspectos negativos, como la corrupción y los privilegios excesivos. Además, aunque el esquema Nosotros está más presente en el discurso, ambos tienden a ser utilizados en conjunto en buena parte de las intervenciones.

Este resultado pone de manifiesto la tendencia que existe en el discurso de López Obrador a presentar una visión dicotómica que contribuye a aumentar la polarización política en el país. Una idea ya apuntada por autores como Marini (2019) o Moreno (2019), pero que en este estudio queda sustentada a partir de los resultados empíricos usados. Ahora bien, más allá de la evidencia acerca de la construcción de la polarización en México que se observa a través de este estudio, los resultados también contribuyen a la comprensión acerca del uso político de las claves periféricas y etiquetas para la construcción del mensaje. En consonancia con lo apuntado por Cho et al. (2006) o Northup et al. (2013), los hallazgos del análisis confirman la existencia de una convergencia de claves periféricas en el discurso, que se realiza de forma natural por el actor político y con la que se contribuye a la creación de una opinión pública.

Además, y en línea con ideas propuestas por autores como Acree et al. (2020), los resultados también sugieren que los actores fuera de procesos electorales tienden a utilizar en su discurso en mayor medida claves periféricas con una fuerte carga ideológica con capacidad de polarizar la sociedad. Este estudio abre una vía de investigación interesante en línea con la teoría de la moderación retórica propuesta recientemente por Acree et al. (2020), que sostiene que los candidatos tienden a moderar sus posiciones durante los procesos electorales debido a una fuerza centrífuga que los lleva a utilizar claves periféricas menos ideologizadas buscando impactar a un electorado más diverso, mientras que fuera de estas etapas se produce una fuerza centrípeta que lleva a los políticos a polarizar su discurso mediante el uso de claves periféricas con alta carga ideológica. El hecho de que el estudio no se haya desarrollado durante un contexto electoral impide conocer hasta qué punto esta diferente estrategia discursiva se presentó en el caso de López Obrador, algo que deberá ser evaluado en futuros estudios. 


\section{REFERENCIAS BIBLIOGRÁFICAS}

Acree, Brice D. L., Justin H. Gross, Noah A. Smith, Yanchuan Sim, y Amber E. Boydstun. 2020. «Etch-a-sketching: Evaluating the post-primary rhetorical moderation hypothesis». American Politics Research, 8.1; 99-131. https://doi.org/10.1177/1532673X18800017

Amezcua Yépiz, Melissa. 2019. «Construcción del líder populista: dos episodios mexicanos paradigmáticos, Lázaro Cárdenas y Andrés Manuel López Obrador». dDeSignis, 31: 157-169. http://dx.doi.org/10.35659/designis.i31p157-169

Carragee, Kevin M. y Wim Roefs. 2004. «The neglect of power in recent framing research». Journal of Communication, 54-2: 214-233. https://doi.org/10.1111/j.1460-2466.2004.tb02625.x

Chaguaceda, Armando. 2019. "Mexico: Political change and delegative democracy». Users Working Paper (Series 2019: 21). The Varieties of Democracy Institute. Disponible en www.v-dem.net

Cho, Jaeho, Homero Gil de Zuniga, Dhavan V. Shah y Douglas M. McLeod. 2006. «Cue convergence. Associative effects on social intolerance». Communication Research, 33.3: 136-154. http://dx.doi.org/10.1177/0093650206287075

D’Angelo, Paul y Donna Shaw. 2018. «Journalism as framing». En Handbook of communication science (pp. 205-233), ed. Tim Vos. Alemania: M. de Gruyter. https://doi.org/10.1515/9781501500084

de Vreese, Claes H. 2012. «New avenues for framing research». American Behavioral Scientist, 56. 3: 365-375. https://doi.org/10.1177/0002764211426331

Druckman, James N. 2001. «The implications of framing effects for citizen competence». Political Behavior, 23.3: 225-256. https://doi.org/10.1023/A:1015006907312

Druckman, James N., Cari Lynn Hennessy, Kristi St. Charles y Jonathan Webber. 2010. «Competing rhetoric over time: Frames versus cues». The Journal of Politics, 72.1: 136-148. https://doi.org/10.1017/S0022381609990521

Edelman, Murray. 1993. «Contestable categories and public opinion». Political Communication, 10.3: 231-242. https://doi.org/10.1080/10584609.1993.9962981

Esteinou, Javier. 2019. «El nuevo gobierno de izquierda y el cambio del modelo de comunicación en México». Revista Internacional de Comunicación y Desarrollo, 10: 122-132. http://dx.doi.org/10.15304/ricd.3.10.6172

Hänglli, Regula. 2012. «Key Factors in frame building: How strategic political actors shape news media coverage». American Behavioral Scientist, 56.3: 300-317. http://dx.doi.org/10.1177/0002764211426327

Hernández Trejo, Ninfa Elizabeth. 2020. «La histórica elección presidencial de 2018». Revista Mexicana de Ciencias Políticas y Sociales, 65.238: 397-402. http://dx.doi.org/10.22201/fcpys.2448492xe.2020.238.69396

Hertog, James, y Douglas M. McLeod. 2001. «A multiperspectival approach to framing analysis: A field guide». In Framing public life (pp. 139-16), eds. Stephen D., Oscar Gandy y August E. Grant. Mahwah, NJ: Erlbaum.

Igartua, Juan José. 2006. Métodos cuantitativos de investigación en comunicación. Barcelona: Bosch.

Igartua, Juan José y Lifen Cheng. 2009. «Moderating effect of group cue while processing news on immigration: Is the framing effect a heuristic process?». Journal of Communication, 59.4: 726-749. https://doi.org/10.1111/j.1460-2466.2009.01454.x

Keum, Heejo, Elloitt D. Hillback, Hernando Rojas, Homero Gil De Zuniga, Dhavan V. Shah y Douglas M. McLeod. 2005. «Personifying the radical: How news framing 
polarizes security concerns and tolerance judgments». Human Communication Research, 3.3: 337-364. https://doi.org/10.1111/j.1468-2958.2005.tb00875.x

Lang, Gladys Engel y Kurt Lang. 1981. «Watergate: An exploration of the AgendaBuilding process». En Mass communication review yearbook 2 (pp. 447-468), eds. Wilhoit G. Cleveland y Harold De Bock. Newbury Park, CA: Sage.

Marini, Anna Marta. 2019. «El mesías tropical: aproximación a fenómenos populistas actuales a través del discurso de López Obrador». Chasqui. Revista Latinoamericana de Comunicación, 139: 153-170. https://doi.org/10.16921/chasqui.v0i139.3665

Martínez Garza, Francisco Javier y Ana Laura Maltos. 2019. «La elección federal en telediarios públicos». Revista Mexicana de Opinión Pública, 14.27: 79-93. https://doi.org/10.22201/fcpys.24484911e.2019.27.68549

Matthes, Jörg. 2012. «Framing politics: An integrative approach». American Behavioral Scientist, 56.3: 247-259. https://doi.org/10.1177/0002764211426324

Mazzoleni, Gianpietro. 2010. La comunicación política. Madrid: Alianza Editorial.

Moreno, Alejandro. 2019. «Introducción: Landslide 2018». En El viraje electoral: opinión pública y voto en las elecciones de 2018 (pp. 25-53), coords. Alejandro Moreno, Alexandra Uribe, y Sergio C. Wals. México: Centro de Estudios Sociales y de Opinión Pública.

Muñiz, Carlos. 2020. «El framing como proyecto de investigación: una revisión de los conceptos, ámbitos y métodos de estudio». Profesional de la Información, 29.6: e290623. https://doi.org/10.3145/epi.2020.nov.23

Nelson, Thomas E. 2004. «Policy goals, public rhetoric, and political attitudes». The Journal of Politics, 66.2: 581-605. https://doi.org/10.1111/j.14682508.2004.00165.x

Northup, Temple y Francesca Dillman Carpentier. 2013. «Exploring priming effectiveness within news stories according to media modality and valence». The Electronic Journal of Communication, 23.3. Recuperado de: http://www.cios.org/EJCPUBLIC/023/3/023032.html

Picazo Zamarripa, Iván David, Aixchel Cordero-Hidalgo y Claudia Patricia Contreras. 2019. «La retórica como esfuerzo deliberado para influir en el ánimo de la sociedad: estudio de casos en los ámbitos científico, político y redes sociales». En Discurso mediático y audiencias: una aproximación crítica a la comunicación de masas (pp. 135-149), ed. Mayte Donstrup. Sevilla: Ediciones Egregius.

Reese, Stephen D. 2007. «The framing project: A bridging model for media research revisited». Journal of Communication, 57.1: 148-154. https://doi.org/10.1111/j.1460-2466.2006.00334.x

Rodelo, Frida V. y Carlos Muñiz. 2019. «Government frames and their influence on news framing: An analysis of cross-lagged correlations in the Mexican context». Global Media and Communication, 15.1: 103-119. http://dx.doi.org/10.1177/1742766518818862

Rotboim, Aviad, Arnon Hershkovitz y Eddie Laventman. 2019. «Positive vs. negative framing of scientific information on Facebook using peripheral cues: An eye tracking study of the credibility assessment process». Interdisciplinary Journal of e-Skills and Lifelong Learning, 15: 81-103. https://doi.org/10.28945/4369

Sánchez Gudiño, Hugo. 2020. «López Obrador, Trump y los mariachis callaron: Manejo de crisis y narrativa de comunicación política de la IV T en México». Agenda Politica, 8.2: 7-26. https://doi.org/10.31990/agenda.2020.2.1 
Sánchez Talanquer, Mariano. 2020. «México 2019: política personalista y neoliberalismo desde la izquierda». Revista de Ciencia Política, 40.2: 401-430. https://doi.org/10.4067/S0718-090X2020005000113

Scheufele, Bertram T. 2006. «Frames, schemata, and news reporting». Communications: The European Journal of Communication Research, 31: 65-83. https://doi.org/10.1515/COMMUN.2006.005

Shah, Dhavan V., Mark D. Watts, David Domke, David P. Fan. 2002. «News framing and cueing of issue regimes: Explaining Clinton's public approval in spite of scandal». Public Opinion Quarterly, 66.3: 339-370. https://doi.org/10.1086/341396

Valentino, Nicholas A., Vincent L. Hutchings y Ismail K. White. 2012. «Cues that matter: How political ads prime racial attitudes during campaigns». American Political Science Review, 96.1: 75-90. https://doi.org/10.1017/S0003055402004240

Villanueva Ulfgard, Rebecka y César Villanueva. 2020. «The power to transform? Mexico's 'Fourth Transformation' under President Andrés Manuel López Obrador». $\quad$ Globalizations, 17.6: 1027-1042. https://doi.org/10.1080/14747731.2020.1718846 